\title{
Self-consistent Simulations of Plasma-Neutral in a Partially Ionized Astrophysical Turbulent Plasma
}

\author{
Dastgeer Shaikh'and G. P. Zank \\ Department of Physics and Center for Space Plasma and Aeronomy Research (CSPAR), \\ The University of Alabama in Huntsville, Huntsville, AL 35899, USA
}

\begin{abstract}
A local turbulence model is developed to study energy cascades in the heliosheath and outer heliosphere $(\mathrm{OH})$ based on self-consistent two-dimensional fluid simulations. The model describes a partially ionized magnetofluid $\mathrm{OH}$ that couples a neutral hydrogen fluid with a plasma primarily through charge-exchange interactions. Charge-exchange interactions are ubiquitous in warm heliospheric plasma, and the strength of the interaction depends largely on the relative speed between the plasma and the neutral fluid. Unlike small-length scale linear collisional dissipation in a single fluid, charge-exchange processes introduce channels that can be effective on a variety of length scales that depend on the neutral and plasma densities, temperature, relative velocities, charge-exchange cross section, and the characteristic length scales. We find, from scaling arguments and nonlinear coupled fluid simulations, that charge-exchange interactions modify spectral transfer associated with large-scale energy-containing eddies. Consequently, the turbulent cascade rate prolongs spectral transfer among inertial range turbulent modes. Turbulent spectra associated with the neutral and plasma fluids are therefore steeper than those predicted by Kolmogorov's phenomenology. Our work is important in the context of the global heliospheric interaction, the energization and transport of cosmic rays, gamma-ray bursts, interstellar density spectra, etc. Furthermore, the plasma-neutral coupling is crucial in understanding the energy dissipation mechanism in molecular clouds and star formation processes.
\end{abstract}

Keywords: MHD Plasma, Neutrals, Charge exchange, Simulations, Space Plasmas

PACS: $96.50 . \mathrm{Ci}$, 96.50.Tf, 96.50.Ya, 96.50.Zc

\section{INTRODUCTION}

In many circumstances, astrophysical and helioshperic plasmas are characterized by partially ionized gases in which magnetized plasma of protons/electrons and neutral particles co-exist. Number densities of these components and interaction processes can vary. The plasma and the neutral particles especially in the heliospheric plasma interact mutually through charge exchange, i.e., a proton sufficiently near a neutral hydrogen atom can capture its electron, thereby creating a new neutral and a proton. This process conserves plasma and neutral densities, but not momentum and energy. The charge exchange mean free path in the local heliospheric plasma is typically about the order of $50 \mathrm{AU}[1]$. Length-scales smaller than the charge exchange mean free path can exhibit turbulent motion. The physics of small-scale turbulent motions is complex and is important to e.g. heating, the transport of cosmic rays, Fermi acceleration, the density spectrum. However no self-consistent simulation models attempt to directly address the multi-component and multiplescale character of the outer heliosphere and its associated turbulence. Here we present a self-consistent plasmaneutral turbulence simulation model based on Fourier spectral techniques that are widely employed in the stud-

${ }^{1}$ Email:dastgeer.shaikh@uah.edu ies of neutral and plasma fluids. We concentrate on some of the most fundamental aspects of partially ionized heliospheric plasma turbulence. In section 2 , we discuss the equations of the coupled plasma-neutral fluid. Section 3 describes results of nonlinear self-consistent fluid simulations that evolve plasma and neutral fluids in the presence of charge exchange forces. The charge exchange spectra are discussed in section 4. Conclusions are presented in section 5 .

\section{PLASMA NEUTRAL COUPLED FLUID MODEL}

Our model simulates the partially ionized plasma in a local region. The fluid model describing nonlinear turbulent processes, in the presence of charge exchange, can be cast into plasma density $\left(\rho_{p}\right)$, velocity $\left(\mathbf{U}_{p}\right)$, magnetic field $(\mathbf{B})$, pressure $\left(P_{p}\right)$ components according to the conservative form

$$
\frac{\partial \mathbf{F}_{p}}{\partial t}+\nabla \cdot \mathbf{Q}_{p}=\mathscr{Q}_{p, n}
$$

where,

$\mathbf{F}_{p}=\left[\begin{array}{c}\rho_{p} \\ \rho_{p} \mathbf{U}_{p} \\ \mathbf{B} \\ e_{p}\end{array}\right], \mathbf{Q}_{p}=\left[\begin{array}{c}\rho_{p} \mathbf{U}_{p} \\ \rho_{p} \mathbf{U}_{p} \mathbf{U}_{p}+\frac{P_{p}}{\gamma-1}+\frac{B^{2}}{8 \pi}-\mathbf{B B} \\ \mathbf{U}_{p} \mathbf{B}-\mathbf{B} \mathbf{U}_{p} \\ e_{p} \mathbf{U}_{p}-\mathbf{B}\left(\mathbf{U}_{p} \cdot \mathbf{B}\right)\end{array}\right]$, 


$$
\mathscr{Q}_{p, n}=\left[\begin{array}{c}
0 \\
\mathbf{Q}_{M}\left(\mathbf{U}_{p}, \mathbf{V}_{n}, \rho_{p}, \rho_{n}, T_{n}, T_{p}\right) \\
0 \\
Q_{E}\left(\mathbf{U}_{p}, \mathbf{V}_{n}, \rho_{p}, \rho_{n}, T_{n}, T_{p}\right)
\end{array}\right]
$$

and

$$
e_{p}=\frac{1}{2} \rho_{p} U_{p}^{2}+\frac{P_{p}}{\gamma-1}+\frac{B^{2}}{8 \pi}
$$

The above set of plasma equations is supplemented by $\nabla \cdot \mathbf{B}=0$ and is coupled self-consistently to the neutral density $\left(\rho_{n}\right)$, velocity $\left(\mathbf{V}_{n}\right)$ and pressure $\left(P_{n}\right)$ through a set of hydrodynamic fluid equations,

$$
\frac{\partial \mathbf{F}_{n}}{\partial t}+\nabla \cdot \mathbf{Q}_{n}=\mathscr{Q}_{n, p}
$$

where,

$$
\begin{gathered}
\mathbf{F}_{n}=\left[\begin{array}{c}
\rho_{n} \\
\rho_{n} \mathbf{V}_{n} \\
e_{n}
\end{array}\right], \mathbf{Q}_{n}=\left[\begin{array}{c}
\rho_{n} \mathbf{V}_{n} \\
\rho_{n} \mathbf{V}_{n} \mathbf{V}_{n}+\frac{P_{n}}{\gamma-1} \\
e_{n} \mathbf{V}_{n}
\end{array}\right], \\
\mathscr{Q}_{n, p}=\left[\begin{array}{c}
\mathbf{Q}_{M}\left(\mathbf{V}_{n}, \mathbf{U}_{p}, \rho_{p}, \rho_{n}, T_{n}, T_{p}\right) \\
Q_{E}\left(\mathbf{V}_{n}, \mathbf{U}_{p}, \rho_{p}, \rho_{n}, T_{n}, T_{p}\right)
\end{array}\right], \\
e_{n}=\frac{1}{2} \rho_{n} V_{n}^{2}+\frac{P_{n}}{\gamma-1} .
\end{gathered}
$$

Equations (11) to (2) form an entirely self-consistent description of the coupled plasma-neutral turbulent fluid. The charge-exchange momentum sources in the plasma and the neutral fluids, i.e. Eqs. (1) and (2), are described respectively by terms $\mathbf{Q}_{M}\left(\mathbf{U}_{p}, \mathbf{V}_{n}, \rho_{p}, \rho_{n}, T_{n}, T_{p}\right)$ and $\mathbf{Q}_{M}\left(\mathbf{V}_{n}, \mathbf{U}_{p}, \rho_{p}, \rho_{n}, T_{n}, T_{p}\right)$. A swapping of the plasma and the neutral fluid velocities in this representation corresponds, for instance, to momentum changes (i.e. gain or loss) in the plasma fluid as a result of charge exchange with the ISM neutral atoms (i.e. $\mathbf{Q}_{M}\left(\mathbf{U}_{p}, \mathbf{V}_{n}, \rho_{p}, \rho_{n}, T_{n}, T_{p}\right)$ in Eq. (1)). Similarly, momentum change in the neutral fluid by virtue of charge exchange with the plasma ions is indicated by $\mathbf{Q}_{M}\left(\mathbf{V}_{n}, \mathbf{U}_{p}, \rho_{p}, \rho_{n}, T_{n}, T_{p}\right)$ in Eq. (2). For a complete description of charge exchange forces, the readers can refer to our work in Ref. [2].

\section{Simulations}

A two-dimensional (2D) nonlinear fluid code was developed to numerically integrate Eqs. (1) to (2). The spatial discretization in our code uses a discrete Fourier representation of turbulent fluctuations based on a pseudospectral method [3], while we use a Runge Kutta 4 method for the temporal integration. All the fluctuations are initialized isotropically (no mean fields are assumed) with random phases and amplitudes in Fourier space. This algorithm ensures conservation of total energy and mean fluid density per unit time in the absence of charge exchange and external random forcing. Additionally, $\nabla \cdot \mathbf{B}=0$ is satisfied at each time step. Our code is massively parallelized using Message Passing Interface (MPI) libraries to facilitate higher resolution. The 2D simulations are not only computationally simpler and less expensive (compared with the full 3D), they offer significantly higher resolutions even on moderate size small cluster machines like Beowulf. Since we are interested in investgating the inertial range spectra, we restrict our simulations to $2 \mathrm{D}$. Our simulations however retain all three components of the magnetic field and the background field is assumed along the y-direction. This enables us to treat the magnetic field appropriately The initial isotropic turbulent spectrum of fluctuations is chosen to be close to $k^{-2}$ (where $k$ is characteristic mode) with random phases in both $x$ and $y$ directions. The choice of such (or even a flatter than -2) spectrum does not influence the dynamical evolution as the final state in our simulations progresses towards fully developed turbulence. While the turbulence code is evolved with time steps resolved self-consistently by the coupled fluid motions, the nonlinear interaction time scales associated with the plasma $1 /\left(\mathbf{k} \cdot \mathbf{U}_{p}(\mathbf{k})\right)$ and the neutral $1 /\left(\mathbf{k} \cdot \mathbf{V}_{n}(\mathbf{k})\right)$ fluids can obviously be disparate. Accordingly, turbulent transport of energy in the plasma and the neutral ISM fluids takes place on distinctively separate time scales.

Spectral transfer in partially ionized fluid turbulence progresses under the action of nonlinear interactions as well as charge exchange sources. Energy cascades amongst turbulent eddies of various scale sizes and between the plasma and the neutral fluids. In a freely decaying case, plasma and neutral fluids evolve under the influence of charge exchange forces which dramatically affect the energy cascades in the inertial range. This is evident from Fig. (1) where Kolmogorov-like [5, 4, 6] fully developed turbulent spectra in the inertial range are shown respectively for the coupled neutral and plasma fluids. The neutral fluid energy spectrum in the inertial regime for the coupled system exhibits a $k^{-\alpha}$ spectrum, where the spectral index $\alpha \approx 3$.2. On the other hand, the spectral index for plasma magnetic and kinetic energy spectra for the coupled system is $\alpha \approx 2.4$. The inertial range spectral indices for the coupled plasma-neutral system in our simulations show a significant deviation from their corresponding uncoupled analogues which are respectively -3 and -1.7 for the neutral and plasma fluids. The spectral indices observed in our simulations can be followed from Kolmogorov-like phenomenology [5, 4, 6], as described in the following.

The typical nonlinear interaction time-scale $\left(\tau_{n l}\right)$ in ordinary (i.e. uncoupled) plasma and/or neutral turbulence 

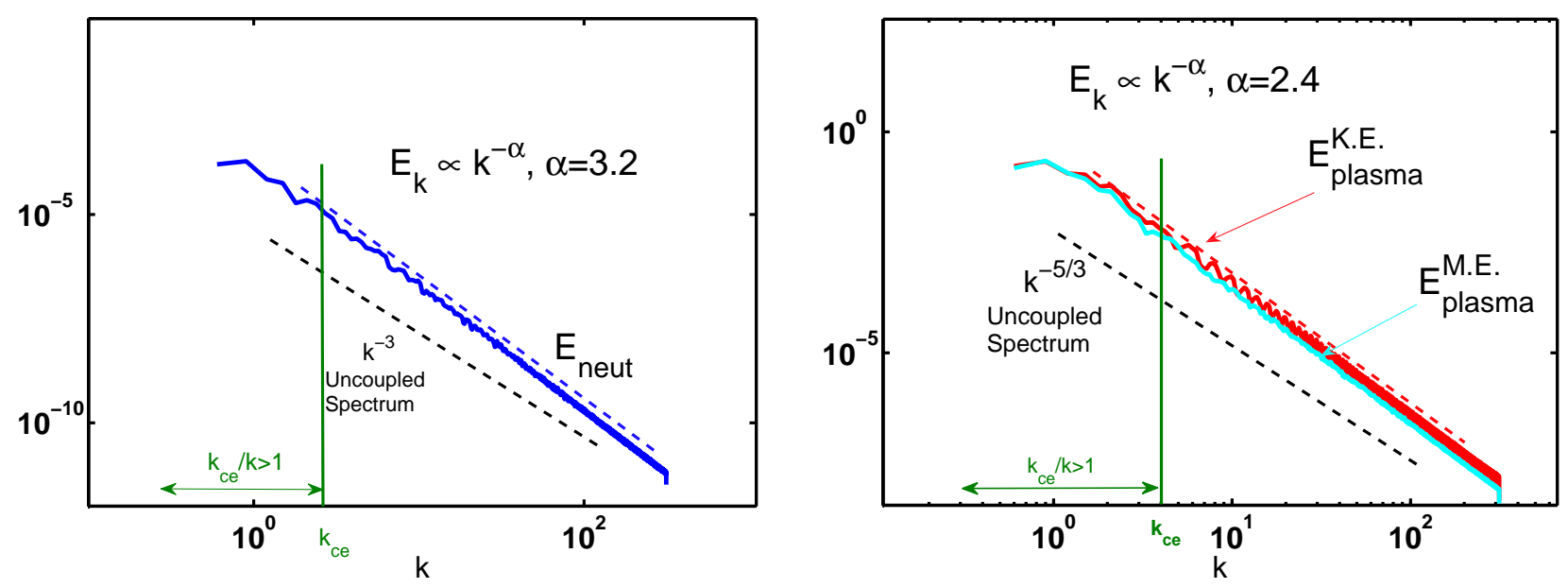

FIGURE 1. (LEFT) Spectra of neutral fluid. (RIGHT) Spectra of plasma fluid.

is given by

$$
\tau_{n l} \sim \frac{\ell_{0}}{v_{\ell}} \sim\left(k v_{k}\right)^{-1},
$$

where $v_{k}$ or $v_{\ell}$ is the velocity of turbulent eddies. In the presence of charge exchange interactions, the ordinary nonlinear interaction time-scale of fluid turbulence is modified by a factor $k_{c e} / k$ such that the new nonlinear interaction time-scale $\left(\tau_{N L}\right)$ in the partially ionized heliospheric turbulence is now

$$
\tau_{N L} \sim \frac{k_{c e}}{k} \frac{1}{k v_{k}} .
$$

On using the fact that $k_{c e}$ is typically larger than $k$ (or $k_{c}$, the characteristic turbulent mode, as defined elsewhere in the paper), i.e. $k_{c e} / k>1$ in the heliosphere [7, 8, 1], the new nonlinear time is $k_{c e} / k$ times bigger than the old nonlinear time i.e. $\tau_{N L} \sim\left(k_{c e} / k\right) \tau_{n l}$. This enhanced nonlinear interaction time in the partially ionized plasma is likely to prolong turbulent energy cascade rates. It is because of this enhanced or prolonged interaction time that a relatively large spectral transfer of turbulent modes tends to steepen the inertial range turbulent spectra in both plasma and neutral fluids. By extending the above phenomenological analysis, one can deduce exact (analytic) spectral indices of the inertial range decaying turbulent spectra, as follows. The new nonlinear interaction time-scale of coupled plasma-neutral turbulence can be rearranged as

$$
\tau_{N L} \sim \frac{k_{c e} v_{k}}{k v_{k}} \frac{1}{k v_{k}} \sim \frac{\tau_{n l}^{2}}{\tau_{c e}}
$$

where $\tau_{c e} \sim\left(k_{c e} v_{k}\right)^{-1}$ represents the charge exchange time scale. The energy dissipation rate $(\varepsilon)$ associated with the coupled plasma-neutral system can be determined from $\varepsilon \sim E_{k} / \tau_{N L}$, which leads to

$$
\varepsilon \sim \frac{v_{k}^{2}}{k_{c e} / k^{2} v_{k}} \sim \frac{k^{2} v_{k}^{3}}{k_{c e}},
$$

where $E_{k}$ is turbulent energy per unit mode. According to the Kolmogorov theory, the spectral cascades are local in $k$-space and the inertial range energy spectrum depends upon the energy dissipation rates and the characteristic turbulent modes, such that $E_{k} \sim \varepsilon^{\gamma} k^{\beta}$. Upon substitution of the above quantities and equating the power of identical bases, one obtains

$$
E_{k} \sim \varepsilon^{2 / 3} k^{-7 / 3}
$$

for the plasma spectrum (the forward cascade inertial range). The spectral index associated with this spectrum, i.e. $7 / 3 \approx 2.33$, is consistent with the plasma spectrum observed in the (coupled plasma-neutral) simulations (see Fig. (1)). Similar arguments in the context of neutral fluids, when coupled with the plasma fluid in the heliosphere, lead to the energy dissipation rates

$$
\varepsilon \sim \frac{k^{2} v_{k}^{2}}{k_{c e} / k^{2} v_{k}} .
$$

This further yields the forward cascade (neutral) energy spectrum

$$
E_{k} \sim \varepsilon^{2 / 3} k^{-11 / 3}
$$

which is close to the simulation result shown in Fig. (1).

\section{CHARGE EXCHANGE PROCESSES}

A key issue finally is to understand what role charge exchange modes play in coupled plasma-neutral helio- 


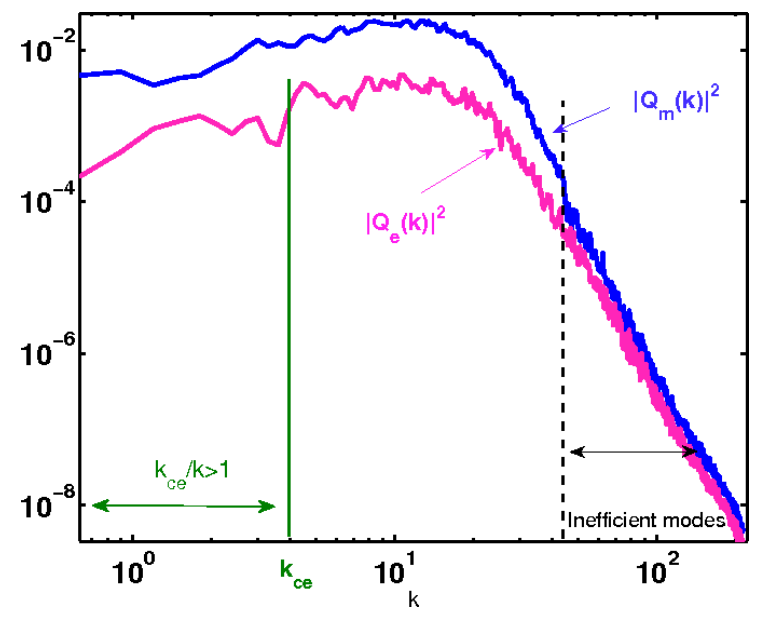

FIGURE 2. Charge exchange spectra

spheric turbulence as they result essentially from the nonlinear charge exchange interactions. To address this issue, we plot charge exchange sources associated with the momentum and energy equations, Eqs. (1) \& (2), in Fig. (2). It appears from Fig. (2) that spectral energy is transferred predominantly at the larger scales by means of charge exchange mode coupling processes. The latter couples the large-scales, or smaller than $k_{c e}$ modes, efficiently to low- $k$ turbulent modes. It is primarily because of this $k_{c e}-k$ mode-coupling in the smaller $k$ part of the spectrum of the coupled plasma-neutral turbulence, that energy is pumped efficiently at the lower $k$ inertial range turbulent modes. The efficient coupling of the Fourier modes at low $k$ 's further enhances the nonlinear eddy time-scales associated with the coupled plasma-neutral turbulence system which is consistent with the scaling $\tau_{N L} \sim\left(k_{c e} / k\right) \tau_{n l}$, where $k_{c e} / k>1$, as described above. This consequently leads to the steepening of the inertial range spectra observed in Fig. (1). By contrast, higher $k$ modes, far from the energy cascade inertial range, are notably inefficient in transferring energy and momentum via charge exchange mode coupling interactions and are damped by small-scale dissipative processes in the coupled plasma-neutral heliospheric turbulence.

\section{CONCLUSIONS}

We have developed a self-consistent fluid model to describe nonlinear turbulent processes in a partially ionized and magnetized heliospheric gas. The charge exchange interactions couple the plasma and the neutral fluids by exciting a characteristic charge exchange coupling mode $k_{c e}$, which is different from the characteristic turbulent mode $k$ of the coupled system. One of the most important points to emerge from our studies is that charge ex- change modes modify the heliospheric turbulence cascades dramatically by enhancing nonlinear interaction time-scales on large scales. Thus on scales $\ell \geq \ell_{c e}$, the coupled plasma system evolves differently than the uncoupled system where large-scale turbulent fluctuations are strongly correlated with charge-exchange modes and they efficiently behave as driven (by charge exchange) energy containing modes of heliospheric turbulence. By contrast, small scale turbulent fluctuations are unaffected by charge exchange modes which evolve like the uncoupled system as the latter becomes less important near the larger $k$ part of the turbulent spectrum. The neutral fluid, under the action of charge exchange, tends to enhance the cascade rates by isotropizing the turbulence on a relatively long time scale. This tends to modify the characteristics of heliospheric turbulence which can be significantly different from the Kolmogorov phenomenology of fully developed turbulence. We believe that, it is because of this enhanced nonlinear eddy interaction time, that a large spectral transfer of turbulent energy tends to smear the current sheets in the magnetic field fluctuations and further cascade energy to the lower Fourier modes in the inertial range turbulent spectra. Consequently it leads to a steeper power spectrum. It is to be noted that the present model does not consider an external driving mechanism, hence the turbulence is freely decaying. Driven turbulence, such as due to large scale external forcing, e.g. supernova explosion, may force turbulence at larger scales. This can modify the cascade dynamics in a manner usually described by dual cascade process.

The support of NASA(NNG-05GH38) and NSF (ATM-0317509) grants is acknowledged.

\section{REFERENCES}

1. G. P. Zank, Sp. Sci. Rev., 89, 413-688 (1999).

2. D. Shaikh, and G. P. Zank, ApJ., 688, 683 (2008).

3. D. Gottlieb and S. A. Orszag, Numerical Analysis of Spectral Methods, SIAM, Philadelphia, (1977).

4. P. S. Iroshnikov, Astron. Zh. 40, 742 (1963).

5. A. N. Kolmogorov, Dokl. Acad. Sci. URSS 30, 301 (1941).

6. R. H. Kraichnan, Phys. Fluids 8, 1385 (1965).

7. V. Florinski, G. P. Zank, and N. V. Pogorelov, J. Geophys. Res., 108(A6), 1228, (2003).

8. V. Florinski, G. P. Zank, and N. V. Pogorelov, J. Geophys. Res., 110, A07104, (2005). 